\title{
Ulcus cruris venosum und ulzerierte Malignome der Unterschenkel - Differenzialdiagnostische Überlegungen am Beispiel eines ulzerierten Leiomyosarkoms
}

G. Wagner

Primary Cutaneous Leiomyosarcoma Clinically Appearing as a Venous Ulceration of the Lower Leg

\section{Zusammenfassung}

Innerhalb weniger Monate entwickelte sich bei dem hier vorgestellten Patienten ein flächenhaft ulzeriertes kutanes Leiomyosarkom an der Streckseite des rechten Unterschenkels. Die ambulante Behandlung war zunächst unter der Diagnose eines Ulcus cruris venosum durchgeführt worden. Zum Zeitpunkt der Klinikeinweisung musste dann eine bereits eingetretene Metastasierung festgestellt werden. Ulzerierte Malignome der Haut können an den Unterschenkeln das klinische Bild eines Ulcus cruris venosum imitieren und sich somit auch längere Zeit der Diagnose entziehen. Bei unserem Patienten waren die Anamnese, die klinische Morphologie des Befundes und das Fehlen typischer Zeichen einer chronisch-venösen Insuffizienz ausschlaggebend für die weiterführende, die Diagnose letztendlich klärende histopathologische Untersuchung.

\section{Abstract}

In this case report a patient is presented with an extensive ulcerous cutaneous leiomyosarcoma which developed at the front of the right lower leg within a few months. This ulceration initially appeared as a venous ulceration and, accordingly an outpatient therapy was initiated. When the patient was admitted to the hospital metastases were already present. Ulcerous malignant skin tumours of the lower legs may clinically imitate a venous ulceration and therefore may not be diagnosed in appropriate time. In our patient case history, clinical morphology as well as missing signs of chronic venous insufficiency led to the definite diagnosis by histopathological examination.

\section{Einleitung}

Unabhängig von der jeweiligen Pathogenese ist das Ulcus cruris in den Industrienationen eine häufige Diagnose. Auf der Grundlage von insgesamt acht umfangreichen epidemiologischen Untersuchungen in Schweden, England und Australien wurde eine durchschnittliche Prävalenz von $0,29 \%$ berechnet. Je nach Studienaufbau waren bei den einzelnen Untersuchungen Prävalenzen zwischen 0,12 und $0,63 \%$ ermittelt worden [1]. Darüber hinaus finden sich in der Literatur mit 1,0-1,3\% auch deutlich höhere Angaben zur Prävalenz, was sich unter anderem dadurch erklärt, dass bei den entsprechenden Untersuchungen vereinzelt auch anamnestisch vorhandene Ulzera mit erfasst worden sind
[2]. Innerhalb der Gruppe der Unterschenkelulzera ist das Ulcus cruris venosum mit weitem Abstand am häufigsten vertreten. Nach Angabe der Alexander House Group sind je nach ausgewerteter Untersuchung 57-80\% aller Unterschenkelulzera auf eine venöse und 10-25\% auf eine arterielle Erkrankung zurückzuführen [4]. Neben den vaskulären Ursachen gibt es zahlreiche weitere Grunderkrankungen, die sich in seltenen Fällen als Ulcus cruris manifestieren können. Hierzu zählen unter anderem die Vaskulitiden, das Pyoderma gangraenosum, hämatologische Erkrankungen, Infektionen, Arzneimittelnebenwirkungen und kutane Malignome [5]. 
Dabei kann die klinisch-morphologische Abgrenzung zwischen dem Ulcus cruris venosum und den verschiedenen, in Einzelfällen auch ulzerierten Malignomen der Haut besondere Schwierigkeiten bereiten. Diese Einschätzung gilt im Übrigen auch für das seltene Vorkommen der malignen Transformation eines Ulcus cruris venosum [6,7]. Somit ist die Situation bei der pathogenetischen Einordnung der Unterschenkelulzera dadurch gekennzeichnet, dass diese insgesamt häufig vorkommende Erkrankung in den meisten Fällen auf eine venöse Insuffizienz zurückzuführen ist, während andererseits jedoch in seltenen Fällen weitere Differenzialdiagnosen zu berücksichtigen sind, die für die betroffenen Patienten naturgemäß eine ganz andere Bedeutung erlangen können als die venös bedingten Unterschenkelulzera. Schwerwiegende Folgen können sich dann ergeben, wenn ulzerierte Malignome der Unterschenkel zunächst als solche nicht erkannt werden, was durch die nachfolgende Kasuistik verdeutlicht werden soll.

\section{Kasuistik}

\section{Anamnese}

Im Mai 2000 bemerkte der damals 54-jährige Patient eine ohne ersichtlichen Grund entstandene Wunde an der Streckseite des rechten Unterschenkels. Der Befund wurde zunächst von seinem Hausarzt als ein Ulcus cruris venosum angesehen und mit verschiedenen fibrinolytisch wirksamen oder antibiotikahaltigen Salben behandelt. Neben einer zusätzlich verordneten Kompressionstherapie wurde der Befund wiederholt mit einem scharfen Löffel mechanisch gereinigt. Trotz dieser konsequent durchgeführten Behandlungen nahm das vermeintliche Ulcus cruris venosum kontinuierlich an Größe zu, so dass der Patient im November 2000 in die Hautklinik Bremerhaven eingewiesen wurde.

In der weiteren Anamnese ergaben sich im Übrigen keine Hinweise für früher aufgetretene Thrombosen, Phlebitiden oder andere Ursachen bzw. Symptome einer chronisch-venösen Insuffizienz.

\section{Dermatologischer Befund}

Distal an der Streckseite des rechten Unterschenkels fand sich ein ca. 8-12 cm durchmessender, aus konfluierenden Knoten zusammengesetzter, oberflächlich erodierter, leicht blutender und speckig belegter Tumor von derber Konsistenz. Die Randbereiche des Tumors waren umschrieben wallartig aufgeworfen oder zeigten, bedingt durch einzelne Knoten, eine polyzyklische, auf die umgebende Haut übergreifende Begrenzung. Die unmittelbare Umgebung des Tumors zeichnete sich durch bizarr konfigurierte, unscharf begrenzte Hyperpigmentierungen aus, während klinische Zeichen einer chronisch-venösen Insuffizienz nicht beobachtet werden konnten (Abb.1 u. 2).

\section{Histopathologischer Befund}

Probebiopsie aus dem Randbereich des Tumors: Das gesamte Exzisat eingenommen von einer malignen mesenchymalen Neoplasie mit ausgedehnten landkartenförmig begrenzten Tumornekrosen. Die Tumorzellen abschnittsweise spindelzellig oder epitheloid und von erheblicher Zellgröße. Sehr große, häufig vesikuläre Zellkerne mit plumpen, pleomorphen Nukleolen. Relativ

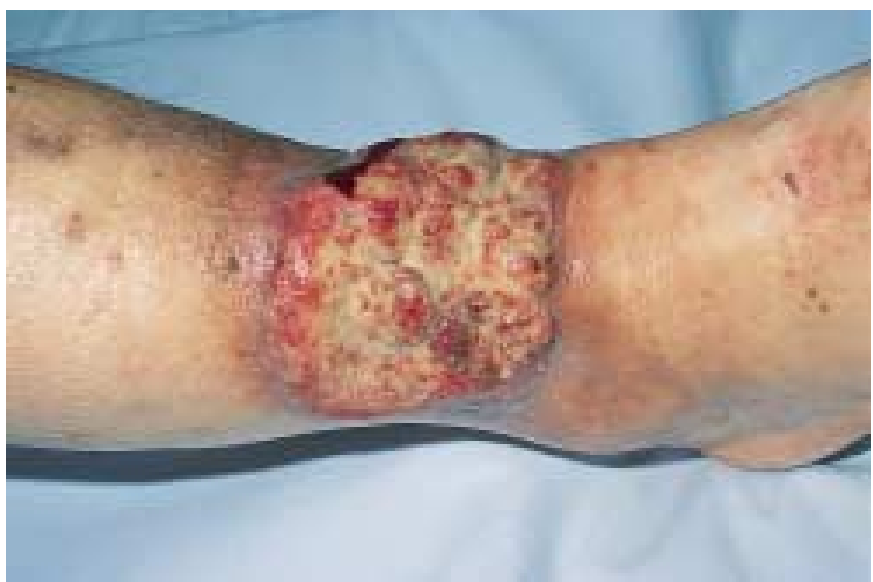

Abb. 1 Ulzeriertes Leiomyosarkom Unterschenkelstreckseite rechts.

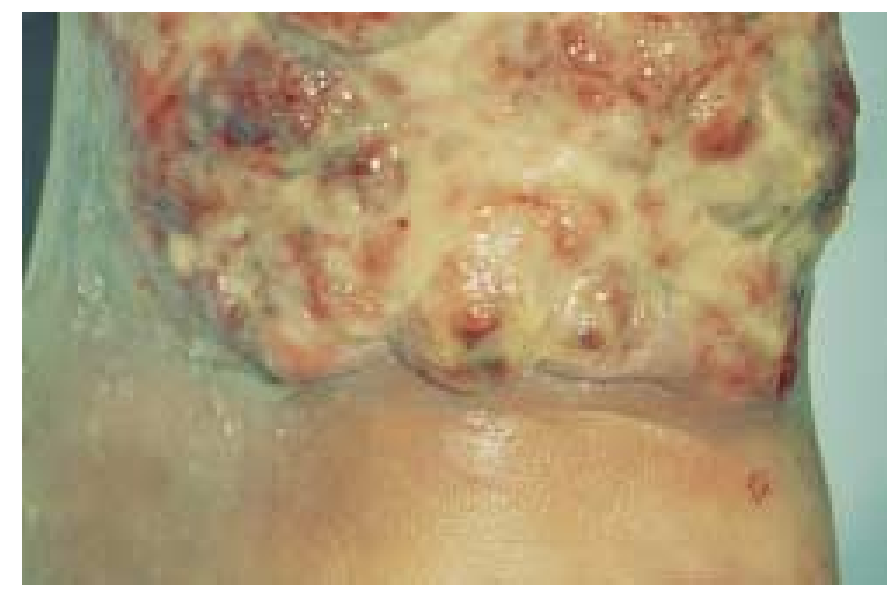

Abb. 2 Detailaufnahme: In die umgebende Haut infiltrativ einwachsende Knoten.

helles, leicht eosinophiles Zytoplasma. In zahlreichen Tumorzellen große zytoplasmatische Vakuolen. Zahlreiche, auch atypische Mitosen.

\section{Immunhistochemie}

Expression der Tumorzellen von Vimentin, Zytokeratin und glattmuskulärem Aktin. Keine Expression von CD 20, CD 30, LCA, EMA und Melan-A. Expression von CD 34 und Faktor VIII-assoziiertes Antigen durch die Gefäßendothelien, nicht jedoch durch die Tumorzellen selbst.

Diagnose: Wenig differenziertes, epitheloides Leiomyosarkom.

\section{Ergänzende Untersuchungen}

Rö Unterschenkel rechts: Subperiostale Verknöcherung der distalen Tibia. Weichteildichte Raumforderung prätibial.

CT/MRT Unterschenkel rechts: Solide Raumforderung prätibial ohne Kontakt zum Knochen. Die solide Periostreaktion oberhalb des Tumors am ehesten durch Lymphabflussstörung bedingt. Der Tumor hat die Faszien zur Tibialis-anterior-Loge infiltriert. Ganzkörperszintigraphie: Fleckige Mehranreicherung distaler Unterschenkel rechts.

Sonographie Leisten: Inguinal rechts mehrere, bis zu $2 \mathrm{~cm}$ durchmessende, sonomorphologisch suspekte Lymphknoten. Rö Thorax und Oberbauchsonographie: Unauffällige Befunde. 
Aufgrund der Ausdehnung des Tumors erfolgte die Unterschenkelamputation in Verbindung mit einer Leistenlymphknotenexstirpation rechts. Die histopathologische Aufarbeitung des Unterschenkels zeigte eine flächenhafte Tumorinfiltration bis in das periostale Weichteilgewebe der Tibia. Darüber hinaus fanden sich Metastasen in zwei von insgesamt zehn exzidierten Lymphknoten. Die empfohlene Lungenteilresektion links lehnte der Patient zunächst ab. Daraufhin erfolgten fünf Zyklen einer Chemotherapie mit Doxorubicin und Ifosfamid. Bei einem anschließenden Re-Staging zeigte sich bedauerlicherweise eine Zunahme der intrapulmonalen Metastasierung. Auch eine dann im September 2001 vorgenommene pulmonale Metastasenresektion konnte den Verlauf der Erkrankung nicht mehr beeinflussen. Der Patient verstarb zwei Monate nach der Thorakotomie.

\section{Diskussion}

$\mathrm{Zu}$ den verschiedenen, auch an den Unterschenkeln gelegentlich in ulzerierter Form auftretenden kutanen Malignomen zählen in der Reihenfolge ihrer Häufigkeit die Basalzellkarzinome, die Melanome und die Plattenepithelkarzinome sowie vereinzelt auch Kaposi-Sarkome, Leiomyosarkome und Lymphome [5, 8,16]. Darüber hinaus kommt es vereinzelt zur malignen Transformation vorbestehender venöser Ulzera oder zur Ausbildung ulzerierter Fistelkarzinome bei chronischer Osteomyelitis $[6,9,10]$. In Einzelfällen müssen die genannten Tumorformen von zahlreichen Unterschenkelulzera anderer Genese, insbesondere jedoch vom Ulcus cruris venosum, differenzialdiagnostisch sicher abgegrenzt werden. Dass diese Forderung keineswegs unproblematisch ist, zeigen verschiedene Kasuistiken, die Patienten mit ulzerierten Malignomen der Unterschenkel beschreiben, die trotz jahrelanger Verläufe zunächst nicht diagnostiziert worden waren [11-13]. Dabei lassen sich verschiedene epidemiologische, anamnestische und klinisch-morphologische Faktoren aufführen, die die Problematik der differenzialdiagnostischen Abklärung zwischen einem Ulcus cruris venosum und den verschiedenen ulzerierten Malignomen der Unterschenkel verdeutlichen. Innerhalb der Gruppe der Unterschenkelulzera ist das Ulcus cruris venosum mit bis zu $80 \%$ am häufigsten vertreten, während ulzerierte Malignome der Unterschenkel ausgesprochen selten sind $[4,8]$. Darüber hinaus zeigen sowohl das Ulcus cruris venosum als auch einzelne ulzerierte Malignome der Unterschenkel epidemiologische Gemeinsamkeiten in ihrer Alters- und Geschlechtsverteilung, so dass die entsprechenden Daten nicht als mögliche Hinweise für die eine oder die andere Diagnose herangezogen werden können. So findet sich das Ulcus cruris venosum nur in Ausnahmefällen vor dem 40. Lebensjahr, um anschließend mit zunehmendem Alter einen kontinuierlichen Anstieg seiner Prävalenz zu zeigen. Dabei sind Frauen in einem Verhältnis von $1: 1,5$ bzw. von $1: 3$ häufiger betroffen als Männer [1]. Diese Situation ist durchaus vergleichbar mit den entsprechenden Daten bei ulzerierten Malignomen der Unterschenkel. In einer von Scholz und Rompel zusammengestellten Statistik der Hautklinik Kassel konnten innerhalb eines Zeitraumes von 20 Jahren 636 Patienten mit Malignomen der Unterschenkel erfasst werden, von denen wiederum 96 Patienten ulzerierte Tumorformen aufwiesen [8]. Innerhalb des Gesamtkollektivs waren Patientinnen sowohl in der Gruppe mit nicht ulzerierten als auch in der Gruppe mit ulzerierten Malignomen häufiger vertreten als die männlichen Patienten. Interessanterweise waren die Patienten mit ulzerierten Malignomen zum Zeitpunkt der Diagnose mit durchschnittlich 68 Jahren älter als die Patienten mit nicht ulzerierten Tumorformen, hatten darüber hinaus eine längere Anamnesedauer und einen größeren Tumordurchmesser, was das Problem der Diagnosestellung bei ulzerierten Malignomen der Unterschenkel noch einmal statistisch unterstreicht.

In der Anamnese der Patienten mit Unterschenkelulzera ergeben sich bisweilen Hinweise, die an ein ulzeriertes Malignom denken lassen sollten. Dabei sind Krankheitsverläufe suspekt, bei denen trotz korrekter Therapie eine kontinuierliche Progression der Ulzera beobachtet werden muss. Auch vollständig fehlende Schmerzen wären für ein Ulcus cruris venosum eher untypisch. Ferner sollte in Zweifelsfällen - z. B. bei ungewöhnlichen Lokalisationen der Ulzera - auf möglicherweise vorbestehende Dermatosen geachtet werden, bei denen erfahrungsgemäß maligne Transformationen vorkommen können. Hierzu zählen am Unterschenkel atrophische Narben, Fisteln bei chronischer Osteomyelitis, das Radioderm, die Morphaea und bekannte Naevuszellnaevi $[9,10,14]$. Die Länge der Anamnese eines Ulcus cruris und die damit verbundene Einschätzung des Größenwachstums lassen hingegen kaum Rückschlüsse auf die jeweilige Dignität zu. Bei ulzerierten Basalzellkarzinomen der Unterschenkel sind Zeiträume von Jahrzehnten beschrieben worden, bis die Ulzerationen handtellergroße Ausdehnungen erreicht hatten, während andererseits $z$. B. Leiomyosarkome - wie auch in dem hier vorgestellten Fall - nur wenige Monate benötigen, um eine vergleichbare Größe zu entwickeln [15 - 17].

Bei der Beurteilung der klinischen Morphologie ist zunächst festzustellen, dass auch bei der Differenzialdiagnose der Unterschenkelulzera keine sicheren Zeichen der Malignität definiert werden können. Das vermutlich wichtigste morphologische Kriterium ist die Tatsache, dass ein ulzeriertes Malignom kein Ulkus im eigentlichen Sinne darstellt, sondern in den meisten Fällen eine knotige Struktur ausbildet, die dann erst sekundär oberflächlich ulzeriert. Dabei bleibt die ursprünglich knotige Struktur häufig vollständig oder umschrieben in den Randbereichen des Tumors erhalten, wobei randständige Knoten infiltrierend in die umgebende Haut einwachsen können [15]. Interessanterweise wurde bei ulzerierten Basalzellkarzinomen der Unterschenkel vereinzelt darauf hingewiesen, dass diese entgegen der allgemeinen Erwartung keineswegs immer einen knotigen Randsaum ausbilden müssen, sondern sich vielmehr flach in die umgebende Haut fortsetzen können $[5,17]$. Dagegen gelten grobknotige, bucklig erscheinende oder verrukös wirkende Granulationsformen besonders in Verbindung mit fehlender Epithelisierungstendenz als weitere Zeichen einer möglicherweise bestehenden Malignität $[6,12,18]$. Das vermeintliche Granulationsgewebe kann sich dabei durch einen auffallend derben Palpationsbefund auszeichnen. Auch spontan oder durch Berührung auslösbare, länger anhaltende Blutungen sollten als suspekt angesehen werden $[5,12]$. Morphologische Besonderheiten einzelner Malignome können sich selbstverständlich auch bei ulzerierten Varianten dieser Tumorformen wiederfinden. So zeigen sich bei ulzerierten Melanomen und ulzerierten Kaposi-Sarkomen häufig noch typische Pigmentierungen in den Randbereichen. 
Ein weiterer morphologischer Hinweis, der bei der diagnostischen Einschätzung eines Ulcus cruris stets berücksichtigt werden sollte, ist der Umstand, dass ein Ulcus cruris venosum immer in Kombination mit anderen klinischen Symptomen der chronisch-venösen Insuffizienz auftritt. Vor dem Hintergrund einer möglichen Koinzidenz schließt allerdings auch das klinische Bild der chronisch-venösen Insuffizienz ein gleichzeitig aufgetretenes ulzeriertes Malignom keineswegs aus [19].

Bei dem hier vorgestellten Patienten fanden sich anamnestisch und klinisch-morphologisch verschiedene Hinweise, die für ein ulzeriertes Malignom sprachen. Dabei ist das kutane Leiomyosarkom sicher eine sehr seltene Differenzialdiagnose zum Ulcus cruris venosum. Die Häufigkeit der Leiomyosarkome wird innerhalb der Gruppe der malignen Weichteiltumoren mit 2,3-5,3\% angegeben [20,21]. Die klinische Morphologie der oberflächlichen Leiomyosarkome ist abhängig vom Ort ihrer Entstehung innerhalb der Hautstrukturen, wobei kutane und subkutane Formen voneinander abgegrenzt werden können [20,22]. Die kutanen, von den $\mathrm{Mm}$. arrectores pilorum abstammenden Leiomyosarkome wachsen langsam und bestehen in der Regel aus derben, miteinander verwachsenen Knoten von rötlicher bis bräunlicher Verfärbung. Hämorrhagische Krusten und Ulzerationen werden als weitere klinische Merkmale angegeben [23,24]. Subkutane Leiomyosarkome, die im Bereich der glatten Muskulatur subkutaner Gefäße entstehen, zeigen sich klinisch als knotige, in der Tiefe lokalisierte Strukturen, wobei sich die Oberfläche der Haut häufig unverändert darstellt. Beide Varianten der oberflächlichen Leiomyosarkome finden sich am häufigsten an den unteren Extremitäten, seltener am Stamm [25,26].

Als Fazit der Kasuistik bleibt abschließend festzustellen, dass die Diagnose eines ulzerierten Malignoms der Unterschenkel letztendlich davon abhängt, ob man im Einzelfall die Möglichkeit eines solchen Geschehens überhaupt in Erwägung zieht. Für die Diagnose entscheidend ist die Durchführung einer histopathologischen Untersuchung.

\section{Literatur}

${ }^{1}$ Wienert V. Neuere epidemiologische Daten zum Unterschenkelgeschwür. Derm 1999; 5: 236-242

2 Ruckley CV. Socioeconomic impact of chronic venous insufficiency and leg ulcers. Angiology 1997; 48: 67-69

${ }^{3}$ Widmer LK. Peripheral venous disorders. Prevalence and sociomedical importance. Basel Study III. Bern: Hans Huber, 1978

${ }^{4}$ The Alexander House Group. Consensus paper on venous leg ulcers. J Dermatol Surg Oncol 1992; 18: 592-602

${ }^{5}$ Miller A, Ruzicka T. Differenzialdiagnose des Ulcus cruris. Hautarzt 2001; 52: 593-603

${ }^{6}$ Wagner G, Knippel H. Ulcus cruris venosum und Plattenepithelkarzinom (Neoplastisches Ulcus cruris). Akt Dermatol 1992; 18: 239-242

${ }^{7}$ Wagner G, Graf L. Plattenepithelkarzinom auf dem Boden eines Ulcus cruris venosum. Akt Dermatol 1999; 25: 117 - 119

${ }^{8}$ Scholz S, Rompel R. Ulzerierte maligne Tumoren als Differenzialdiagnose beim Ulcus cruris. Z Hautkr 2001; 76: 41

${ }^{9}$ Scharnagel E, Smola MG, Hellbom BA, Pierer G, Hoflehner H. Narbenkarzinom: Beobachtungen und Ergebnisse in 23 Fällen. Langenbecks Arch Chir 1991; 376: 341 - 345

10 Wagner G, Graf L, Seiler H. Plattenepithelkarzinom bei posttraumatischer chronischer Osteomyelitis. Akt Dermatol 1998; 24: 314-317

${ }^{11}$ Keilig W, Keilig W. Zwei Basaliome an den Unterschenkeln einer 60jährigen Frau - eines davon unter dem Bild eines Ulcus cruris. Hautarzt 1984; 35: 546-549

12 Mailänder W, Müller RPA. Plattenepithelkarzinom des Unterschenkels - ein interdisziplinäres therapeutischen Vorgehen. Derm 1996; 2: $418-424$

${ }^{13}$ Müller W, Zaun H. Basaliome in Verletzungs- und Operationsnarben. Akt Derm 1981; 7: $37-40$

${ }^{14}$ Laux B, Bräuninger W. Entwicklung eines spinozellulären Karzinoms auf dem Boden einer Morphaea generalisata. Z Hautkr 1985; 60: $767-773$

${ }^{15}$ Breger G, Kingreen JCH. Das „therapieresistente“ Ulcus cruris: Gelegentlich ein Basaliom? Akt Dermatol 1984; 10: 34 - 35

${ }^{16}$ Sauer $\mathrm{CH}$. Kutanes Leiomyosarkom unter dem Bild eines Ulcus cruris. Hautnah Derm 1993; 5: $442-445$

17 Wagner G, Niemeyer U. U. Ulcus cruris venosum oder Ulcus rodens des Unterschenkels - eine nicht alltägliche Differenzialdiagnose. Der Deutsche Dermatologe 1993; 8: 824-826

${ }^{18}$ Harris B, Eaglstein WH, Falanga V. Basal cell carcinoma arising in venous leg ulcers and mimicking granulation tissue. J Dermatol Surg Oncol 1993; 19: 150-153

${ }^{19}$ Kluess HG, Gallenkemper G, Kreysel HW. Epitheliale Tumoren als Differenzialdiagnose des Ulcus cruris - Basaliom und Plattenepithelkarzinom. Phlebol 1994; 23: 193 - 196

${ }^{20}$ Dahl I, Angervall L. Cutaneous and subcutaneous Leiomyosarcoma: a clinicopathologic study of 47 patients. Pathol Eur 1974; 9: 307-315

${ }^{21}$ Stout AP, Hill WT. Leiomyosarcoma of the superficial soft tissues. Cancer 1958; $11: 844-854$

22 Auroy S, Contesso G, Spatz G, Genin J, Margulis A, Lecesne A, Avril MF. Primary cutaneous leiomyosarkoma: 32 cases. Ann Dermatol Venereol 1999; 126: 235-242

${ }^{23}$ Stieler W, Mensing H, Schaeg G, Jehn E. Primäres kutanes Leiomyosarkom. Hautarzt 1991; 42: 44-47

24 Wagner G, Barghorn A. Kutanes Leiomyosarkom. Akt Dermatol 2001; 27: $30-33$

${ }^{25}$ Fields JP, Helwig EB. Leiomyosarcoma of the skin und subcutaneous tissue. Cancer 1981; 47: 156-169

${ }^{26}$ Hashimoto H, Daimaru Y, Tsuneyoshi M, Enjoji M. Leiomyosarcoma of the external soft tissues. A clinicopathologic, immunhistochemical and electron microscopic study. Cancer 1986; 57: 2077-2088 